University of Wollongong

Research Online

Faculty of Engineering and Information

Faculty of Engineering and Information

Sciences - Papers: Part A

Sciences

$1-1-2016$

\title{
Recovery of absolute phases for the fringe patterns of three selected wavelengths with improved anti-error capability
}

Jiale Long

Wuyi University, Huazhong University of Science and Technology

Jiangtao Xi

University of Wollongong, jiangtao@uow.edu.au

Jianmin Zhang

Wuyi University, coolstarinfo@163.com

Ming Zhu

Huazhong University of Science and Technology, zhuming@mail.hust.edu.cn

Wengqing Cheng

Huazhong University of Science and Technology

See next page for additional authors

Follow this and additional works at: https://ro.uow.edu.au/eispapers

Part of the Engineering Commons, and the Science and Technology Studies Commons

Research Online is the open access institutional repository for the University of Wollongong. For further information contact the UOW Library: research-pubs@uow.edu.au 


\title{
Recovery of absolute phases for the fringe patterns of three selected wavelengths with improved anti-error capability
}

\author{
Abstract \\ In a recent published work, we proposed a technique to recover the absolute phase maps of fringe \\ patterns with two selected fringe wavelengths. To achieve higher anti-error capability, the proposed \\ method requires employing the fringe patterns with longer wavelengths; however, longer wavelength may \\ lead to the degradation of the signal-to-noise ratio (SNR) in the surface measurement. In this paper, we \\ propose a new approach to unwrap the phase maps from their wrapped versions based on the use of \\ fringes with three different wavelengths which is characterized by improved anti-error capability and SNR. \\ Therefore, while the previous method works on the two-phase maps obtained from six-step phase-shifting \\ profilometry (PSP) (thus 12 fringe patterns are needed), the proposed technique performs very well on \\ three-phase maps from three steps PSP, requiring only nine fringe patterns and hence more efficient. \\ Moreover, the advantages of the two-wavelength method in simple implementation and flexibility in the \\ use of fringe patterns are also reserved. Theoretical analysis and experiment results are presented to \\ confirm the effectiveness of the proposed method.
}

\section{Keywords}

error, anti, improved, wavelengths, selected, three, capability, patterns, recovery, fringe, phases, absolute

Disciplines

Engineering | Science and Technology Studies

\section{Publication Details}

J. Long, J. Xi, J. Zhang, M. Zhu, W. Cheng, Z. Li \& Y. Shi, "Recovery of absolute phases for the fringe patterns of three selected wavelengths with improved anti-error capability," Journal of Modern Optics, vol. 63, (17) pp. 1695-1705, 2016.

\section{Authors}

Jiale Long, Jiangtao Xi, Jianmin Zhang, Ming Zhu, Wengqing Cheng, Zhongwei Li, and Yusheng Shi 


\section{Recovery of absolute phases for the fringe patterns of three selected wavelengths with improved anti-error capability}

Jiale Long ${ }^{1,2}$, Jiangtao $\mathrm{Xi}^{3 *}$, Jianmin Zhang ${ }^{1}$, Ming Zhu' ${ }^{2}$, Wenqing Cheng ${ }^{2}$, Zhongwei $\mathrm{Li}^{4}$ and Yusheng Shi ${ }^{4}$

${ }^{1}$ School of Information, Wuyi University, Jiangmen, Guangdong, 529020, China,

${ }^{2}$ School of Electronic Information and Communications, Huazhong University of Science and Technology, Wuhan, Hubei, 430074, China

${ }^{3}$ School of Electrical Computer and Telecommunications Engineering, University of Wollongong, Wollongong, NSW, 2522, Australia

${ }^{4}$ State Key Laboratory of Material Processing and Die \& Mould Technology, Huazhong University of Science and Technology, Wuhan, Hubei, 430074, China

Jiale Long: +8613422530782, longjiale_528@126.com

*Corresponding author Jiangtao Xi: +612 42213412, jiangtao@uow.edu.au

Jianmin Zhang: +8613422778736, zjm99_2001 @126.com

Ming Zhu: +8618627810937, zhuming@mail.hust.edu.cn

Wenqing Cheng: +8613807144980, chengwq@mail.hust.edu.cn

Zhongwei Li: +8618627779624, zwli@hust.edu.cn

Yusheng Shi: +8627 87557954, shiyusheng@hust.edu.cn

Acknowledgements: This work was partially supported by the Science Foundation for Young Teachers of Wuyi University under Grant number 2013zk06; Guangdong province innovation project of education department under Grant number 2013KJCX0185; and the National Natural Science Foundation of China under Grant number 61371139. 


\section{Recovery of absolute phases for the fringe patterns of three selected wavelengths with improved anti-error capability}

In a recent published work we proposed a technique to recover the absolute phase maps of fringe patterns with two selected fringe wavelengths. To achieve higher anti-error capability, the proposed method requires employing the fringe patterns with longer wavelengths; however, longer wavelength may lead to the degradation of the signal-to-noise ratio (SNR) in the surface measurement. In this paper, we propose a new approach to unwrap the phase maps from their wrapped versions based on the use of fringes with three different wavelengths which is characterized by improved anti-error capability and SNR. Therefore, while the previous method works on the two phase maps obtained from six-step PSP (thus 12 fringe patterns are needed), the proposed technique performs very well on three phase maps from three steps PSP, requiring only 9 fringe patterns and hence more efficient. Moreover, the advantages of the two-wavelength method in simple implementation and flexibility in the use of fringe patterns are also reserved. Theoretical analysis and experiment results are presented to confirm the effectiveness of the proposed method.

Keywords: phase shift; fringe analysis; phase unwrapping; projected fringes; surface measurement

\section{Introduction}

With the development of digital technology, fringe projection profilometry (FPP) has become one of the most promising technologies for non-contact 3D shape measurement (1-3). To recover absolute phase maps from the wrapped ones, phase unwrapping is a necessary and important step in the implementation of FPP. Due to the complex nature of object surface shape and various unwanted noises inherent to the projection and acquisition of fringe patterns, phase unwrapping is a challenging task. Many approaches have been studied and employed such as spatial (4-7) and temporal (8-13). Spatial phase unwrapping approaches assume a continuity of scanned surfaces and likely fail in regions with depth discontinuities (step edges) (10). Temporal methods use multiple fringe patterns, which recovers the absolute phase on pixel-by-pixel basis, hence not suffering from error propagation between pixels. In order to keep the efficiency of the temporal methods, it is always desirable to employ as less number of images as possible. Zhao, et al. (8) proposed to use the fringe patterns of two wavelengths, one of which has a very long wavelength to cover the whole projection area, and hence can be used as a reference to unwrap the other fringe pattern. $\mathrm{Li}$, et al. (9) also employ a projection image containing a single fringe as a reference to calculate the fringe order of the high spatial frequency fringe patterns. As indicated in (11) long spatial wavelength fringe patterns is disadvantageous by low phase-sensitivity to the object surface shape, short spatial wavelength fringe patterns are always preferred in order to achieve accurate shape measurement. However, if the gap between the frequencies of the two fringe patterns is larger than a certain value, the 
before-mentioned methods would fail due to the existence of noise and disturbance in the fringe patterns, and hence more intermediate fringe patterns should be employed (10). Recently, Servin, et al. (11) proposed a 2-step temporal phase unwrapping formula that uses so-called 2-sensitivity demodulated phases for measuring static surfaces. The first phase demodulation $\phi 1(x, y)$ has at most 1-wavelength sensitivity and the second one $\phi 2(x, y)$ is G-times $(\mathrm{G}>>1)$ more sensitive. However, the phase error defined in the paper is $\phi e(x, y)=[\phi 2(x, y)]-G \phi 1(x, y)]$ which should be limited within $(-\pi, \pi)$. This condition implies a phase error tolerance bound $\pi /(1+G)$ for the method in (11), beyond which the method may not work properly. In such cases, more fringe patterns should still be needed. Hence, minimization of the number of fringe patterns while improving the anti-error capability of phase unwrapping is a key problem for application of temporal approaches in practice.

Ding, et al. (14) proposed a method to unwrap absolute phase maps of fringe patterns with two selected frequencies. The two normalized spatial frequencies defined in (14) are the numbers of fringes on the images which denoted by $f_{1}$ and $f_{2}$, which are assumed to be integers. The performance of the method is limited by phase error tolerance bound, $\pi /\left(f_{1}+f_{2}\right)$ and requirement of frequencies coprime (15). For purpose of increasing phase error tolerance bound, a new approach of absolute phase recovery is presented which based on the use of fringe patterns with three selected spatial frequencies denoted by $f_{1}, f_{2}$ and $f_{3}$, where the frequencies are also assumed to be integers (16).

While the methods proposed in (14-16) provide efficient ways for phase unwrapping, they also suffer from a weakness, that is, the total number of pixels perpendicular to the fringe must be an integer multiple of the number of pixels within a fringe. Such a selection may not be convenient. Taking the experiment described in (16) as an example, the resolution of the projector is $1392 \times 1038$ pixels. If the selected frequencies are $\left(f_{1}, f_{2}, f_{3}\right)=(6,10,15)$ as in $(16)$, the numbers of pixels per fringe period will be $232,139.2$ and 92.8 respectively, which are not integers and thus are not implementable. Furthermore, only when $f_{1}$, $f_{2}$ and $f_{3}$ do not have a common factor larger than 1 , there exists a unique solution for the phase unwrapping problem as proved in (16). Such a selection may not be possible in some cases. For the cases of the horizontal resolution being 1024 pixels, which is common for many ordinary projectors, it is impossible to find three frequencies meeting the requirement. A possible solution to this problem is to tailor the whole image to a smaller size, which may lead to the degradation of resolution in the 3D measurement which we have analyzed in (17).

In order to solve the above mentioned problem, in the recent published work (17) we proposed a technique to recover the absolute phase maps of two sets of fringe patterns with flexible selection of fringe wavelengths (denoted by $\lambda_{1}$ and $\lambda_{2}$ ). It should be noted that wavelength as defined in this paper is different from that defined in physics. The wavelength here represents the total number of pixels per fringe period. We have proved that when we get the wrapped phase maps by the six-step phase-shifting profilometry (PSP), the absolute phase maps can be recovered correctly. We also have proved that the method in (14) is a special case of the approach proposed in (17). That is, when $\lambda_{1}$ and $\lambda_{2}$ are co-prime, 
and the number of fringes on the images are integers, the conclusion in (14) tallies with the conclusion in (17). The allowed range of phase error in (17) is larger than that in (14) and the same with which in (16) when $\lambda_{1}$ and $\lambda_{2}$ are not co-prime. Furthermore, the computation in (17) is much less.

However, we realized that the anti-error capability as measured by phase error tolerance bound of the proposed technique depends on the greatest common divisor (g.c.m.) of the two fringe wavelengths (denoted by $k$ ), that is the bigger the $k$ is, the better the anti-error capability can get. Let $\lambda_{1}=k g_{1}$ and $\lambda_{2}=k g_{2}$ where $g_{1}$ and $g_{2}$ are positive integers which are co-prime, the upper bound of the allowable phase error is $k \pi /\left(\lambda_{1}+\lambda_{2}\right)=\pi /\left(g_{1}+g_{2}\right)$, if better anti-error capability is desired, $g_{1}+g_{2}$ should be decreased. But at the same time, the validity of the method relies on $R \leq \lambda_{1} \lambda_{2} / k=g_{1} g_{2} k$, where $R$ is the resolution of

projector, which means once the projection is fixed, $k$ should be increased as $g_{1}$ and $g_{2}$ decreased, and this will resulted in a multiple growth of the selected wavelengths. Taking the experiment described in (17) as an example, the vertical resolution of the projector is 768 pixels, as the maximal phase error on the wrapped phase maps which get from six-step PSP is about $\pi / 100$, two fringe wavelengths $\left(\lambda_{1}, \lambda_{2}\right)$ meeting the criteria is easy to select, such as $(23,47),(52,100)$. However, when the maximal phase error on the wrapped phase maps which get from three-step PSP increases to about $\pi / 10$, the selected wavelengths of the fringes should be very long, such as $(156,195),(159,265)$. As we know, for a phase-shifting technique, the longer the wavelength, the lower the phase sensitivity to the object shape. In other words, short wavelength should be used to achieve high SNR associated with the fringe patterns for accurate phase unwrapping. Therefore, it is worthwhile to study if there is a room for further improvement in the antierror capability.

In this paper, we try to extend the method proposed in (17) to the cases of fringe patterns with three different wavelengths. The three wavelengths $\lambda_{1}, \lambda_{2}$ and $\lambda_{3}$ are still positive integers representing the total number of pixels in a fringe period. We will demonstrate that the anti-error capability can be considerably improved without increasing the wavelengths of fringe patterns. In addition, the absolute phase maps can still be recovered correctly for the wrapped phase maps from the three-step PSP. Therefore, with suitable selections of fringe wavelengths, only 9 fringe patterns are needed, which is more efficient than the method proposed in (17).

The paper is organized as follows. Section 2 presents the phase unwrapping algorithm with three sets of fringe patterns. In Section 3, we give the principle for fringe wavelengths selection. The phase error bound is derived in Section 4. In Section 5, experiments and results are presented to validate the proposed method. Finally we conclude the paper in Section 6.

\section{Absolute phase map recovery of fringe patterns with three difference wavelengths}

Consider that three sets of sinusoidal fringe patterns are projected onto the surface of an object, which are reflected and captured by a camera. These fringe patterns of vertical resolution $\mathrm{R}$ pixels are with three different wavelengths, denoted by $\lambda_{1}, \lambda_{2}$ and $\lambda_{3}$ (in pixels) respectively. The projected image can be 
expressed as follows:

$$
I_{p i}(x, y)=a_{p}(x, y)+b_{p}(x, y) \cos \left[\Phi_{p i}(y)\right](i=1,2,3), y \in[0, R)
$$

where $(x, y)$ are the coordinates of a pixel in the projector and camera images, and $\Phi_{p i}(y)=\frac{2 \pi}{\lambda_{i}} y$ is the absolute phase of the projected image (or the carrier). The image reflected from the object and captured by the camera (denoted as camera image) can be expressed as

$$
I_{c i}(x, y)=a_{c}(x, y)+b_{c}(x, y) \cos \left[\Phi_{c i}(y)\right](i=1,2,3), y \in[0, R)
$$

where $\Phi_{c i}(y)=\Phi_{p i}(y-u(y))$, and $u(y)$ is spatial shift caused by the object surface shape described by triangular relationship among the positions of the projector, the camera and the target (12). As $\Phi_{p i}(y)$ is a liner function of $y$, we also have $\Phi_{c i}(y)=\Phi_{p i}(y)+\Delta \Phi_{i}(y)$, where $\Delta \Phi_{i}(y)=-\frac{2 \pi}{\lambda_{i}} u(y)$. Hence, in order to reconstruct the 3D surface of the object, we need $\Delta \Phi_{i}(y)$, which can be obtained by $\Phi_{c i}(y)-\Phi_{p i}(y)$, thus requiring the absolute phase $\Phi_{c i}(y)$. However, all existing methods can only yield $\phi_{c i}(y)$, which are the wrapped version of the absolute phases with their values falling within $[-\pi, \pi)$. The absolute phases $\Phi_{c i}(y)$ are related to the wrapped ones by the following:

$$
\Phi_{c i}(y)=2 \pi m_{i}(y)+\phi_{c i}(y)
$$

where $m_{i}(y)$ are integers and are the fringe order index associated with pixel $(x, y)$.

As mentioned above, $\Phi_{c i}(y)$ are a spatially shifted version of the phase of the carrier fringe patterns $\Phi_{p i}(y)$, and hence there is a unique corresponding mapping relationship between $\Phi_{c i}(y)$ and $\Phi_{p i}(y)$ which purely depends on $u(y)$ or the surface shape. With all the three fringe patterns being projected on the same object, $u(y)$ should be the same in relating $\Phi_{c i}(y)$ and $\Phi_{p i}(y)$, and the same relationship applies for the wrapped versions of $\Phi_{c i}(y)$ and $\Phi_{p i}(y)$ as well. As the temporal approach unwrap the phase on pixel-bypixel basis, the same method can be applied to both the carrier patterns as well as the patterns reflected from the target. In other words, we do not need to consider if these wrapped phases are from the carrier only patterns or these reflected from the object surface. In the following, we will simply work on $\Phi_{c i}(y)$. Without loss of generality, the ranges of $\varphi_{c 1}(y), \varphi_{c 2}(y)$ and $\varphi_{c 3}(y)$ are shifted by $\pi$, yielding the following:

$$
0<\varphi_{c 1}(y), \varphi_{c 2}(y), \varphi_{c 3}(y)<2 \pi
$$

From Eq. (3), we have:

$$
\left\{\begin{array}{l}
\Phi_{c 1}(y)=2 \pi m_{1}(y)+\varphi_{c 1}(y) \\
\Phi_{c 2}(y)=2 \pi m_{2}(y)+\varphi_{c 2}(y) \\
\Phi_{c 3}(y)=2 \pi m_{3}(y)+\varphi_{c 3}(y)
\end{array}\right.
$$

With the help of assuming a pattern with only one fringe covering the whole image with its absolute phase 
$\Phi_{0}(y)$ within $(0,2 \pi)$, we have $\Phi_{c i}(y)=\frac{R}{\lambda_{i}} \Phi_{0}(y)$ (for $\left.i=1,2,3\right)$. Then we are able to obtain the following:

$$
\begin{aligned}
& {\left[\lambda_{1} \varphi_{1}(y)-\lambda_{2} \varphi_{2}(y)\right] / 2 \pi=m_{2}(y) \lambda_{2}-m_{1}(y) \lambda_{1}} \\
& {\left[\lambda_{1} \varphi_{1}(y)-\lambda_{3} \varphi_{3}(y)\right] / 2 \pi=m_{3}(y) \lambda_{3}-m_{1}(y) \lambda_{1}} \\
& {\left[\lambda_{2} \varphi_{2}(y)-\lambda_{3} \varphi_{3}(y)\right] / 2 \pi=m_{3}(y) \lambda_{3}-m_{2}(y) \lambda_{2}}
\end{aligned}
$$

Note that the left hand sides of Eqs.(6) (8) can be obtained by PSP, which must be integers as the right sides are integers. If there are one-to-one correspondences between the right sides and the three integers $m_{1}\left(y_{c}\right), m_{2}\left(y_{c}\right)$ and $m_{3}\left(y_{c}\right)$, Eqs. (6) (8) reveal a way to determine these three integers based on the values of the left hand sides. Combining Eqs.(6) (8) and the expressions (4) we have:

$$
\begin{aligned}
& -\lambda_{2}<\left[\lambda_{1} \varphi_{1}(y)-\lambda_{2} \varphi_{2}(y)\right] / 2 \pi<\lambda_{1}, \text { i.e., }-\lambda_{2}<\left[m_{2}(y) \lambda_{2}-m_{1}(y) \lambda_{1}\right]<\lambda_{1} \\
& -\lambda_{3}<\left[\lambda_{1} \varphi_{1}(y)-\lambda_{3} \varphi_{3}(y)\right] / 2 \pi<\lambda_{1}, \text { i.e., }-\lambda_{3}<\left[m_{3}(y) \lambda_{3}-m_{1}(y) \lambda_{1}\right]<\lambda_{1} \\
& -\lambda_{3}<\left[\lambda_{2} \varphi_{2}(y)-\lambda_{3} \varphi_{3}(y)\right] / 2 \pi<\lambda_{2}, \text { i.e., }-\lambda_{3}<\left[m_{3}(y) \lambda_{3}-m_{2}(y) \lambda_{2}\right]<\lambda_{2}
\end{aligned}
$$

At the same time, from Eqs. (5) and the expressions (4), we have:

$$
\begin{aligned}
& 0 \leq m_{1}(y)<R / \lambda_{1} \\
& 0 \leq m_{2}(y)<R / \lambda_{2} \\
& 0 \leq m_{3}(y)<R / \lambda_{3}
\end{aligned}
$$

With inequalities (9) (14) above, an unique mapping from $\left[\lambda_{1} \varphi_{1}(y)-\lambda_{2} \varphi_{2}(y)\right] / 2 \pi,\left[\lambda_{1} \varphi_{1}(y)-\lambda_{3} \varphi_{3}(y)\right] / 2 \pi$, $\left[\lambda_{2} \varphi_{2}(y)-\lambda_{3} \varphi_{3}(y)\right] / 2 \pi$ to $m_{1}(y), m_{2}(y)$ and $m_{3}(y)$ can be identified.

Now let us to utilize an example to prove the effectiveness of this method, where we choose $\lambda_{1}=54$, $\lambda_{2}=72, \lambda_{3}=90$ and $R=1024$. By varying $m_{1}(y), m_{2}(y)$ and $m_{3}(y)$ over the range defined by Eqs. (12) (14), we are able to obtain $m_{2}(y) \lambda_{2}-m_{1}(y) \lambda_{1}, m_{3}(y) \lambda_{3}-m_{1}(y) \lambda_{1}$ and $m_{3}(y) \lambda_{3}-m_{2}(y) \lambda_{2}$. Then we check the values against the range given by Eqs. (9) (11) and these meeting the conditions can be listed in Table 1.

From Eqs.(5), the whole range of $y_{p}$ (i.e., $\left.[0, R)\right)$ can be separated based on the value of $m_{1}(y), m_{2}(y)$ and $m_{3}(y)$ as follows:

$$
\begin{gathered}
m_{1}(y)= \begin{cases}0, & 0 \leq y_{p}<\lambda_{1} \\
1, & \lambda_{1} \leq y_{p}<2 \lambda_{1} \\
\cdots & \cdots \\
{\left[R / \lambda_{1}\right]-1,} & \lambda_{1}\left(\left[R / \lambda_{1}\right]-1\right) \leq y_{p}<\lambda_{1}\left[R / \lambda_{1}\right] \\
{\left[R / \lambda_{1}\right],} & \lambda_{1}\left[R / \lambda_{1}\right] \leq y_{p}<R\end{cases} \\
m_{2}(y)= \begin{cases}0, & 0 \leq y_{p}<\lambda_{2} \\
1, & \lambda_{2} \leq y_{p}<2 \lambda_{2} \\
\cdots & \cdots \\
{\left[R / \lambda_{2}\right]-1,} & \lambda_{2}\left(\left[R / \lambda_{2}\right]-1\right) \leq y_{p}<\lambda_{2}\left[R / \lambda_{2}\right] \\
{\left[R / \lambda_{2}\right],} & \lambda_{2}\left[R / \lambda_{2}\right] \leq y_{p}<R\end{cases}
\end{gathered}
$$




$$
m_{3}(y)= \begin{cases}0, & 0 \leq y_{p}<\lambda_{3} \\ 1, & \lambda_{3} \leq y_{p}<2 \lambda_{3} \\ \cdots & \cdots \\ {\left[R / \lambda_{3}\right]-1,} & \lambda_{3}\left(\left[R / \lambda_{3}\right]-1\right) \leq y_{p}<\lambda_{3}\left[R / \lambda_{3}\right] \\ {\left[R / \lambda_{3}\right],} & \lambda_{3}\left[R / \lambda_{3}\right] \leq y_{p}<R\end{cases}
$$

Where $[x]$ denotes the largest integer not greater than $x$. As the vertical resolution of projection image is $R=1024$, from Eqs.(15) (17) we can see that the first, second and the third columns of Table 1 cover all the possible values of $m_{1}(y), m_{2}(y)$ and $m_{3}(y)$, and the last three columns meet the requirements of the desired range, that is, $-\lambda_{2}<\left[m_{2}(y) \lambda_{2}-m_{1}(y) \lambda_{1}\right]<\lambda_{1},-\lambda_{3}<\left[m_{3}(y) \lambda_{3}-m_{1}(y) \lambda_{1}\right]<\lambda_{1}$ and $-\lambda_{3}<\left[m_{3}(y) \lambda_{3}-m_{2}(y) \lambda_{2}\right]<\lambda_{2}$ without repetition.

The above example shows that when the three wavelengths of fringe patterns have common factor, the phase maps can be unwrapped. In fact, no matter whether the three wavelengths of phase maps have or do not have common factors, phase unwrapping can always be done by the above mentioned approach. Through above analysis the absolute phase could be retrieved from the wrapped phase maps of fringe patterns with three selected fringe wavelengths by the following steps.

(1) Select three fringe wavelengths $\left(\lambda_{1}, \lambda_{2}, \lambda_{3}\right)$ using the criteria described in Section 3 to ensure the unique mapping from $m_{2}(y) \lambda_{2}-m_{1}(y) \lambda_{1}, m_{3}(y) \lambda_{3}-m_{1}(y) \lambda_{1}, m_{3}(y) \lambda_{3}-m_{2}(y) \lambda_{2}$ to $m_{1}(y), m_{2}(y), m_{3}(y)$ and construct a lookup table like Table 1;

(2) Project the three sets of fringe patterns onto the object and obtain the unwrapped phase maps $\varphi_{1}(y)$, $\varphi_{2}(y)$ and $\varphi_{3}(y)$;

(3) Calculate $\left[\lambda_{1} \varphi_{1}(y)-\lambda_{2} \varphi_{2}(y)\right] / 2 \pi,\left[\lambda_{1} \varphi_{1}(y)-\lambda_{3} \varphi_{3}(y)\right] / 2 \pi,\left[\lambda_{2} \varphi_{2}(y)-\lambda_{3} \varphi_{3}(y)\right] / 2 \pi$ and round them to the nearest integers. Find the row of the table constructed in step 1 whose value of $m_{2}(y) \lambda_{2}-m_{1}(y) \lambda_{1}$, $m_{3}(y) \lambda_{3}-m_{1}(y) \lambda_{1}, m_{3}(y) \lambda_{3}-m_{2}(y) \lambda_{2}$ is closest to the integers. Keep the records of $m_{1}(y), m_{2}(y)$ and $m_{3}(y)$ in the same row;

(4) Retrieve the absolute phase maps by Eqs.(5) using $m_{1}(y), m_{2}(y)$ and $m_{3}(y)$ acquired in step 3.

The process of creating the lookup table does not need to take $\Phi_{0}(y)$ which increases monotonically from $-\pi$ to $\pi$ as the reference to analyze the interval distribution of the fringe orders like in (16), and hence does not need to do the interval partition at all. As only the simple inequations need to be checked, so, the computation is much less in compared to the amount required in (16).

\section{Selection of the three fringe wavelengths}

From the analysis in appendix, there exists an unique mapping from $\left(m_{1}(y), m_{2}(y), m_{3}(y)\right)$ to $\left[\lambda_{1} \varphi_{1}(y)-\lambda_{2} \varphi_{2}(y)\right] / 2 \pi,\left[\lambda_{1} \varphi_{1}(y)-\lambda_{3} \varphi_{3}(y)\right] / 2 \pi$ and $\left[\lambda_{2} \varphi_{2}(y)-\lambda_{3} \varphi_{3}(y)\right] / 2 \pi$ (i.e., $m_{2}(y) \lambda_{2}-m_{1}(y) \lambda_{1}$, 
$\left.m_{3}(y) \lambda_{3}-m_{1}(y) \lambda_{1}, m_{3}(y) \lambda_{3}-m_{2}(y) \lambda_{2}\right)$ when $R \leq \lambda_{1} \lambda_{2} \lambda_{3} /\left(k^{2} k_{1} k_{2} k_{3}\right)$, where $R$ is the resolution of projector, and $k$ is the g.c.m. of $\lambda_{1}, \lambda_{2}$ and $\lambda_{3}, \lambda_{1}=k g_{1}, \lambda_{2}=k g_{2}, \lambda_{3}=k g_{3}$, where $k_{1}$ is the g.c.m. of $g_{1}$ and $g_{2}, k_{2}$ is the g.c.m. of $g_{1}$ and $g_{3}, k_{3}$ is the g.c.m. of $g_{2}$ and $g_{3}$. That is, the constraint of the selection of three fringe wavelengths is:

$$
R \leq \lambda_{1} \lambda_{2} \lambda_{3} /\left(k^{2} k_{1} k_{2} k_{3}\right)
$$

\section{Phase error bound}

When we round the values of $\left[\lambda_{1} \varphi_{1}(y)-\lambda_{2} \varphi_{2}(y)\right] / 2 \pi,\left[\lambda_{1} \varphi_{1}(y)-\lambda_{3} \varphi_{3}(y)\right] / 2 \pi$ and $\left[\lambda_{2} \varphi_{2}(y)-\lambda_{3} \varphi_{3}(y)\right] / 2 \pi$ to the nearest integers and find the row of the table constructed in step 1 with the values of $m_{2}(y) \lambda_{2}-m_{1}(y) \lambda_{1}$, $m_{3}(y) \lambda_{3}-m_{1}(y) \lambda_{1}$ and $m_{3}(y) \lambda_{3}-m_{2}(y) \lambda_{2}$ closest to the integers, three values are required to match, which should follow a proper order. For example, if $\left[\lambda_{1} \varphi_{1}(y)-\lambda_{2} \varphi_{2}(y)\right] / 2 \pi$ (i.e., the value of fourth column) is the one to match first, and $\left[\lambda_{1} \varphi_{1}(y)-\lambda_{3} \varphi_{3}(y)\right] / 2 \pi$ (i.e., the value of fifth column) follows, the anti-error capability of the proposed technique first depends on the smallest gaps between any two possible value of $m_{2}(y) \lambda_{2}-m_{1}(y) \lambda_{1}$, and then $m_{3}(y) \lambda_{3}-m_{1}(y) \lambda_{1}$. The larger the gaps, the less likely the error will happen during the rounding operations.

In (17), we have proved that the minimal gap of $m_{2}(y) \lambda_{2}-m_{1}(y) \lambda_{1}$ must be equal or greater than $k$, where $k$ is the g.c.m. of $\lambda_{1}$ and $\lambda_{2}$. Then, we can deduce that the smallest gap between the value of $m_{2}(y) \lambda_{2}-m_{1}(y) \lambda_{1}$ is $k_{1}$ which is the g.c.m of $\lambda_{1}$ and $\lambda_{2}$, and the smallest gap between the value of $m_{3}(y) \lambda_{3}-m_{1}(y) \lambda_{1}$ is $k_{2}$ which is the g.c.m of $\lambda_{1}$ and $\lambda_{3}$, the smallest gap between the value of $m_{3}(y) \lambda_{3}-m_{2}(y) \lambda_{2}$ is $k_{3}$ which is the g.c.m of $\lambda_{2}$ and $\lambda_{3}$.

Hence, for achieving higher anti-error capability, the first column to match should be the biggest one of $k_{1}, k_{2}$ and $k_{3}$, and the last column to match should be the smallest one. From Table 1, it is easy to discover that if two columns have been matched, the third one surely matched.

Assuming phase errors in the phase maps $\varphi_{1}(y), \varphi_{2}(y)$ and $\varphi_{3}(y)$ are $\Delta \varphi_{1}(y), \Delta \varphi_{2}(y)$ and $\Delta \varphi_{3}(y)$

respectively, and $\Delta \varphi_{\max }=\max \left(\left|\Delta \varphi_{1}(y)\right|,\left|\Delta \varphi_{2}(y)\right|,\left|\Delta \varphi_{3}(y)\right|\right)$, we have:

$$
\left\{\begin{array}{l}
{\left[\lambda_{1} \Delta \varphi_{1}(y)-\lambda_{2} \Delta \varphi_{2}(y)\right] / 2 \pi<\left(\lambda_{1}+\lambda_{2}\right) \Delta \varphi_{\max } / 2 \pi<k_{1} / 2} \\
{\left[\lambda_{1} \Delta \varphi_{1}(y)-\lambda_{3} \Delta \varphi_{3}(y)\right] / 2 \pi<\left(\lambda_{1}+\lambda_{3}\right) \Delta \varphi_{\max } / 2 \pi<k_{2} / 2} \\
{\left[\lambda_{2} \Delta \varphi_{2}(y)-\lambda_{3} \Delta \varphi_{3}(y)\right] / 2 \pi<\left(\lambda_{2}+\lambda_{3}\right) \Delta \varphi_{\max } / 2 \pi<k_{3} / 2}
\end{array}\right.
$$

Then we can find the upper bound of the allowable phase error, with which the absolute phase maps can be correctly recovered:

$$
\left\{\begin{array}{l}
0 \leq \Delta \varphi_{\max }<k_{1} \pi /\left(\lambda_{1}+\lambda_{2}\right) \\
0 \leq \Delta \varphi_{\max }<k_{2} \pi /\left(\lambda_{1}+\lambda_{3}\right) \\
0 \leq \Delta \varphi_{\max }<k_{3} \pi /\left(\lambda_{2}+\lambda_{3}\right)
\end{array}\right.
$$

Note that, only two columns should be matched in this method, and so, the allowable phase error should 
be change to:

$$
0 \leq \Delta \varphi_{\max }<\operatorname{median}\left[k_{1} \pi /\left(\lambda_{1}+\lambda_{2}\right), k_{2} \pi /\left(\lambda_{1}+\lambda_{3}\right), k_{3} \pi /\left(\lambda_{2}+\lambda_{3}\right)\right]
$$

In another word, if $k_{1}<k_{2}<k_{3}$, we can choose the two columns whose minimal gap are $k_{2}$ and $k_{3}$ to match only, then the allowable phase error changes to $\left\{\begin{array}{l}0 \leq \Delta \varphi_{\max }<k_{2} \pi /\left(\lambda_{1}+\lambda_{3}\right) \\ 0 \leq \Delta \varphi_{\max }<k_{3} \pi /\left(\lambda_{2}+\lambda_{3}\right)\end{array}\right.$, i.e., $0 \leq \Delta \varphi_{\max }<\min \left(k_{2} \pi /\left(\lambda_{1}+\lambda_{3}\right), k_{3} \pi /\left(\lambda_{2}+\lambda_{3}\right)\right)$.

If $\Delta \varphi_{\max }$ is given, the three fringe wavelengths should be selected to meet two of the following three conditions at least.

$$
\left\{\begin{array}{l}
\left(\lambda_{1}+\lambda_{2}\right)<k_{1} \pi / \Delta \varphi_{\max } \\
\left(\lambda_{1}+\lambda_{3}\right)<k_{2} \pi / \Delta \varphi_{\max } \\
\left(\lambda_{2}+\lambda_{3}\right)<k_{3} \pi / \Delta \varphi_{\max }
\end{array}\right.
$$

For example, suppose the resolution of the projection image is $R=1024$, if we want to unwrap the wrapped phase maps obtained from three-step PSP whose maximal phase error is about $\pi / 10$, the fringe wavelengths could be chosen as $\left(\lambda_{1}, \lambda_{2}, \lambda_{3}\right)=(54,72,90)$ whose phase error tolerance bound is $0 \leq \Delta \varphi_{\max }<\pi / 8$. If the method proposed in (17) is employed, a pair of $\left(\lambda_{1}, \lambda_{2}\right)$ which have large g.c.m should be selected, e.g., $(208,260)$ whose phase error tolerance bound is $0 \leq \Delta \varphi_{\max }<\pi / 9$. If the method proposed in (16) is used, the selected frequencies are $\left(f_{1}, f_{2}, f_{3}\right)=(8,12,15)$ whose phase error tolerance bound is $0 \leq \Delta \varphi_{\max }<\pi / 9$ as described in (16), and the corresponding wavelengths are $\left(\lambda_{1}, \lambda_{2}, \lambda_{3}\right)=(128,85.3,68.3)$. Hence for the same level of phase error tolerance, we can choose much shorter wavelength for the proposed method than that in (16) and (17). Considering that the fringe patterns with long wavelengths suffer from low SNR in the phase maps, the method proposed is advantageous by high SNR in the phase maps and hence accurate 3D measurement.

\section{Experiments}

Experiments are carried out to test the proposed method with a system consisting of a LG HW300 projector and Daheng HV1351 camera, with their resolutions being $768 \times 1024$ and $1024 \times 1280$ respectively. The object to be measured is a toy model which is shown in Fig.1. Firstly, three sets of sinusoidal fringe patterns with fringe wavelengths of 54, 72 and 90 are projected onto the toy model, as shown in Fig. 2(a), 2(b) and 2(c). Note that the vertical resolution of projector is 1024, i.e., $R=1024$. The wrapped phase maps obtained from three-step PSP are shown in Fig. 2(d), 2(e) and 2(f). Since the background does not contain useful information, a shadow noise detector/filter $(18,19)$ was employed which is shown in Fig. 3(a), such that the shadow-noised regions were discarded from further processing (19). The maximal phase error on the wrapped phase maps is about $\pi / 10$, which is smaller than median $\left(k_{1} \pi /\left(\lambda_{1}+\lambda_{2}\right), k_{2} \pi /\left(\lambda_{1}+\lambda_{3}\right), k_{3} \pi /\left(\lambda_{2}+\lambda_{3}\right)\right)=\pi / 8$. Hence based on Eq.(21) the absolute phase maps can be successfully recovered. Fig. 3(b) is the unwrapped phase map of $\lambda=54$ which is characterized by monotonic variance over the areas of smooth shape change on the model, and hence results are considered 
as correct. The anti-error capability is considerably increased in contrast to (17) and the SNR can be improved due to the use of the fringe patterns with short wavelengths.

Secondly, three sets of fringe patterns with different wavelengths of 25, 30 and 35 are projected onto the same toy model, as shown in Fig. 4(a), 4(b) and 4(c). Four-step PSP is used and thus leads to lower level of noise in the wrapped phase maps, as shown in Fig.4(d), 4(e) and 4(f). The shadow noise detector/filter is also employed in Fig. 5(a). The maximal phase error on the wrapped phase maps is about $\pi / 15$, which is smaller than the error bound $\pi / 13$. Hence based on Eq.(21) the absolute phase maps can be successfully recovered. Fig.5(b) is the unwrapped phase map of $\lambda=25$ which is characterized by monotonic variance over the areas of smooth shape change on the model, and hence results are considered as correct. The anti-error capability and the SNR here are both improved incontrast to the performance of (17). In order to see the topographic details of the object clearly, we remove the carrier-plane from Fig.5(b) and get Fig.5(c). The 3D view of the recovered object is shown in Fig.6.

\section{Conclusion}

This paper proposes a new approach to recover the absolute phase maps based on wrapped ones of the fringe patterns with three selected wavelengths. Compared with our previous work in (17), the anti-error capability is increased for reliable phase unwrapping implementation and the SNR can be improved for the use of shorter wavelengths. With suitable selections of fringe wavelengths, the absolute phase maps can be correctly recovered using only 9 fringe patterns, which is more efficient than the method proposed in (17). Moreover, in comparison with the existing method in (16), the proposed method is advantageous by less computation required for constructing the checking tables and more flexibility in the design of fringe patterns. The effectiveness of the proposed method has been verified by experimental results.

\section{References:}

(1) Christopher J., Waddington, Jonathan D. Kofman. Opt. Eng. 2014, 53, 084109.

(2) Haixia Wang, Qian Kemao, Seah Hock Soon. Opt. Exp. 2015, 23, 7535-7549.

(3) Z.H.Zhang. Opt Lasers Eng. 2012, 50, 1097-1106.

(4) S. Zhang, X. Li, S.-T.Yau. Appl. Opt. 2007, 46, 50-57.

(5) S. Li, W. Chen, X. Su. Appl. Opt. 2008, 47, 3369-3377.

(6) Y. Shi. Opt. Exp. 2007, 15, 8059-8064.

(7) M. Costantini. IEEE Trans. Geoscience and Remote Sensing. 1998, 36, 813-821.

(8) H. Zhao, W. Chen, Y. Tan. Appl. Opt.1994, 33, 4497-4500.

(9) J. Li, L. G. Hassebrook, C. Guan. J. Opt. Soc. Am. A. 2003, 20, 106-115.

(10) J. M. Huntley, H. O. Saldner. J. Opt. Soc. Am. A. 1997, 14, 3188-3196.

(11) M. Servin, J.M. Padilla, A. Gonzalez, G. Garnica. Opt. Exp. 2015, 23, 15806-15815.

(12) Y. Hu, J. Xi, J. Chicharo, E. Li, Z. Yang. Appl. Opt. 2006, 45, 6560 6567.

(12) G. Pedrini, I. Alexeenko, W. Osten, H. J. Tiziani. Appl. Opt. 2003, 42, 5846-5854.

(13) Liu Yong, Huang Dingfa, Jiang Yong. Appl. Opt. 2012, 51, 4945-4953. 
(14) Y. Ding, J. Xi, Y. Yu, J. Chicharo. Opt. Let. 2011, 36, 2518-2520.

(15) Y. Ding, J. Xi, Y. Yu, W. Q. Cheng, S. Wang, J. Chicharo. Opt. Exp. 2012, 20, 13238-13251.

(16) Y. Ding, J. Xi, Y. Yu, F. Deng. Opt. Laser. Eng. 2015, 70, 18-15.

(17) J. Long, J. Xi , M. Zhu, W. Cheng, R. Cheng, Z. Li, Y. Shi. Appl. Opt. 2014, 53, 1794-1801.

(18) X. Su, G. von Bally, D. Vukicevic. Opt. Commun. 1993, 98, 141-150.

(19) K. Liu, Y. Wang, D. L. Lau, Q. Hao, L. G. Hassebrook. Opt. Exp. 2010, 18, 5229-5244.

\section{Appendix: Analysis on selection of the three fringe wavelengths}

The validity of this method relies on the existence of unique mapping from $m_{2}(y) \lambda_{2}-m_{1}(y) \lambda_{1}$, $m_{3}(y) \lambda_{3}-m_{1}(y) \lambda_{1}, m_{3}(y) \lambda_{3}-m_{2}(y) \lambda_{2}$ to $m_{1}(y), m_{2}(y), m_{3}(y)$. This requires that both sides of Eqs.(6) (8) must not yield the same value for any two different pixel number index such as $y_{a}$ and $y_{b}(a \neq b)$. In other words, the following must hold:

$$
\left\{\begin{array}{l}
{\left[\lambda_{2} m_{2}\left(y_{a}\right)-\lambda_{1} m_{1}\left(y_{a}\right)\right] \neq\left[\lambda_{2} m_{2}\left(y_{b}\right)-\lambda_{1} m_{1}\left(y_{b}\right)\right]} \\
{\left[\lambda_{3} m_{3}\left(y_{a}\right)-\lambda_{1} m_{1}\left(y_{a}\right)\right] \neq\left[\lambda_{3} m_{3}\left(y_{b}\right)-\lambda_{1} m_{1}\left(y_{b}\right)\right]} \\
{\left[\lambda_{3} m_{3}\left(y_{a}\right)-\lambda_{2} m_{2}\left(y_{a}\right)\right] \neq\left[\lambda_{3} m_{3}\left(y_{b}\right)-\lambda_{2} m_{2}\left(y_{b}\right)\right]}
\end{array}\right.
$$

From Table 1 we can see that if the first two equations hold, the last one surely holds. So, without loss of generality, Eqs.(23) can be simplified to Eqs.(24). Let us first discuss the simpler case where $\lambda_{1}, \lambda_{2}$ and $\lambda_{3}$ are coprime with each other.

$$
\left\{\begin{array}{l}
{\left[\lambda_{2} m_{2}\left(y_{a}\right)-\lambda_{1} m_{1}\left(y_{a}\right)\right] \neq\left[\lambda_{2} m_{2}\left(y_{b}\right)-\lambda_{1} m_{1}\left(y_{b}\right)\right]} \\
{\left[\lambda_{3} m_{3}\left(y_{a}\right)-\lambda_{1} m_{1}\left(y_{a}\right)\right] \neq\left[\lambda_{3} m_{3}\left(y_{b}\right)-\lambda_{1} m_{1}\left(y_{b}\right)\right]}
\end{array} \quad \text { for } y_{a} \neq y_{b}\right.
$$

The above can be proved by reductio ad absurdum. Suppose there exist $y_{a}$ and $y_{b}(a \neq b)$ making the two side of Eqs.(24) equal, and three possible situations may make the two sets of $\left(m_{1}(y), m_{2}(y), m_{3}(y)\right)$ different: firstly, the three pairs of integers are all different with each other, i.e., $m_{1}\left(y_{a}\right) \neq m_{1}\left(y_{b}\right)$, $m_{2}\left(y_{a}\right) \neq m_{2}\left(y_{b}\right)$ and $m_{3}\left(y_{a}\right) \neq m_{3}\left(y_{b}\right)$; secondly, there are two pairs of integers different, such as $m_{1}\left(y_{a}\right)=m_{1}\left(y_{b}\right), m_{2}\left(y_{a}\right) \neq m_{2}\left(y_{b}\right)$ and $m_{3}\left(y_{a}\right) \neq m_{3}\left(y_{b}\right)$; thirdly, only one pair of them is different, such as $m_{1}\left(y_{a}\right)=m_{1}\left(y_{b}\right), m_{2}\left(y_{a}\right)=m_{2}\left(y_{b}\right)$ and $m_{3}\left(y_{a}\right) \neq m_{3}\left(y_{b}\right)$.

Without losing generosity, we take the first case into account where $m_{1}\left(y_{a}\right) \neq m_{1}\left(y_{b}\right), m_{2}\left(y_{a}\right) \neq m_{2}\left(y_{b}\right)$, $m_{3}\left(y_{a}\right) \neq m_{3}\left(y_{b}\right)$. When the two sides of (24) equal, we have:

$$
\left\{\begin{array}{l}
{\left[m_{2}\left(y_{a}\right)-m_{2}\left(y_{b}\right)\right] / \lambda_{1}=\left[m_{1}\left(y_{a}\right)-m_{1}\left(y_{b}\right)\right] / \lambda_{2}} \\
{\left[m_{3}\left(y_{a}\right)-m_{3}\left(y_{b}\right)\right] / \lambda_{1}=\left[m_{1}\left(y_{a}\right)-m_{1}\left(y_{b}\right)\right] / \lambda_{3}}
\end{array}\right.
$$

As $\lambda_{1}, \lambda_{2}$ and $\lambda_{3}$ are coprime two by two, Eqs.(25) must be equivalent to the following:

$$
\left\{\begin{array}{l}
{\left[m_{2}\left(y_{a}\right)-m_{2}\left(y_{b}\right)\right]=n_{1} \lambda_{1},\left[m_{1}\left(y_{a}\right)-m_{1}\left(y_{b}\right)\right]=n_{1} \lambda_{2}} \\
{\left[m_{3}\left(y_{a}\right)-m_{3}\left(y_{b}\right)\right]=n_{2} \lambda_{1},\left[m_{1}\left(y_{a}\right)-m_{1}\left(y_{b}\right)\right]=n_{2} \lambda_{3}}
\end{array}\right.
$$

Where $n_{1}, n_{2}$ are integers and $n_{1}, n_{2} \neq 0$. From Eqs.(26) we know: 


$$
n_{1} \lambda_{2}=n_{2} \lambda_{3}
$$

Because $\lambda_{1}, \lambda_{2}$ and $\lambda_{3}$ are coprime two by two, we have $n_{1}=n \lambda_{3}, n_{2}=n \lambda_{2}$, where $n$ is an integer and $n \neq 0$, then: $\left[m_{1}\left(y_{a}\right)-m_{1}\left(y_{b}\right)\right]=n \lambda_{3} \lambda_{2},\left[m_{2}\left(y_{a}\right)-m_{2}\left(y_{b}\right)\right]=n \lambda_{3} \lambda_{1},\left[m_{3}\left(y_{a}\right)-m_{3}\left(y_{b}\right)\right]=n \lambda_{2} \lambda_{1}$. Combining inequations (12) (14), we have : $\quad-R / \lambda_{1}<\left[m_{1}\left(y_{a}\right)-m_{1}\left(y_{b}\right)\right]<R / \lambda_{1},-R / \lambda_{2}<\left[m_{2}\left(y_{a}\right)-m_{2}\left(y_{b}\right)\right]<R / \lambda_{2}$ and $-R / \lambda_{3}<\left[m_{3}\left(y_{a}\right)-m_{3}\left(y_{b}\right)\right]<R / \lambda_{3}$ which means $-R / \lambda_{1}<n \lambda_{2} \lambda_{3}<R / \lambda_{1},-R / \lambda_{2}<n \lambda_{1} \lambda_{3}<R / \lambda_{2}$ and $-R / \lambda_{3}<n \lambda_{2} \lambda_{1}<R / \lambda_{3}$. Hence, Eqs.(23) will hold when $R \leq|n| \lambda_{1} \lambda_{2} \lambda_{3} \leq \lambda_{1} \lambda_{2} \lambda_{3}$,where $|n|$ is the absolute value of $n$.

For the other two cases, it is obvious that the same conclusions will get.

When $\lambda_{1}, \lambda_{2}$ and $\lambda_{3}$ are not coprime, there are two possible situations. Firstly, let $k$ be the greatest common divisor (g.c.m.) of $\lambda_{1}, \lambda_{2}$ and $\lambda_{3}$, we have $\lambda_{1}=k g_{1}, \lambda_{2}=k g_{2}, \lambda_{3}=k g_{3}$ where $g_{1}, g_{2}$ and $g_{3}$ are positive integers which are coprime with each other. Equations (24) can be reproduced as follow:

$$
\left\{\begin{array} { l } 
{ [ k g _ { 2 } m _ { 2 } ( y _ { a } ) - k g _ { 1 } m _ { 1 } ( y _ { a } ) ] \neq [ k g _ { 2 } m _ { 2 } ( y _ { b } ) - k g _ { 1 } m _ { 1 } ( y _ { b } ) ] } \\
{ [ k g _ { 3 } m _ { 3 } ( y _ { a } ) - k g _ { 1 } m _ { 1 } ( y _ { a } ) ] \neq [ k g _ { 3 } m _ { 3 } ( y _ { b } ) - k g _ { 1 } m _ { 1 } ( y _ { b } ) ] }
\end{array} \text { i.e., } \left\{\begin{array}{l}
{\left[g_{2} m_{2}\left(y_{a}\right)-g_{1} m_{1}\left(y_{a}\right)\right] \neq\left[g_{2} m_{2}\left(y_{b}\right)-g_{1} m_{1}\left(y_{b}\right)\right]} \\
{\left[g_{3} m_{3}\left(y_{a}\right)-g_{1} m_{1}\left(y_{a}\right)\right] \neq\left[g_{3} m_{3}\left(y_{b}\right)-g_{1} m_{1}\left(y_{b}\right)\right]}
\end{array}\right.\right.
$$

Obviously Eqs.(28) is the same as Eqs.(24), and hence can be proved using the same approach. That is Eqs.(28) will hold when $R^{\prime} \leq g_{1} g_{2} g_{3}$, where $R^{\prime}=R / k^{2}$, which is equivalent to $R \leq \lambda_{1} \lambda_{2} \lambda_{3} / k^{2}$.

Secondly, when the g.c.m. of $\lambda_{1}, \lambda_{2}$ and $\lambda_{3}$ are not exists, but they have g.c.m. between any two of them. Let $k_{1}$ be the g.c.m. of $\lambda_{1}$ and $\lambda_{2}, \lambda_{1}=k_{1} g_{1}, \lambda_{2}=k_{1} g_{2}$, where $g_{1}, g_{2}$ are positive integers which are coprime; $k_{2}$ be the g.c.m. of $\lambda_{1}$ and $\lambda_{3}, \lambda_{1}=k_{2} h_{1}, \lambda_{3}=k_{2} h_{2}$, where $h_{1}, h_{2}$ are positive integers which are coprime; $k_{3}$ be the g.c.m. of $\lambda_{2}$ and $\lambda_{3}$. Equations (25) can be reproduced as follow:

$$
\left\{\begin{array} { l } 
{ [ k _ { 1 } g _ { 2 } m _ { 2 } ( y _ { a } ) - k _ { 1 } g _ { 1 } m _ { 1 } ( y _ { a } ) ] = [ k _ { 1 } g _ { 2 } m _ { 2 } ( y _ { b } ) - k _ { 1 } g _ { 1 } m _ { 1 } ( y _ { b } ) ] } \\
{ [ k _ { 2 } h _ { 2 } m _ { 3 } ( y _ { a } ) - k _ { 2 } h _ { 1 } m _ { 1 } ( y _ { a } ) ] = [ k _ { 2 } h _ { 2 } m _ { 3 } ( y _ { b } ) - k _ { 2 } h _ { 1 } m _ { 1 } ( y _ { b } ) ] }
\end{array} \text { i.e., } \left\{\begin{array}{l}
{\left[g_{2} m_{2}\left(y_{a}\right)-g_{1} m_{1}\left(y_{a}\right)\right]=\left[g_{2} m_{2}\left(y_{b}\right)-g_{1} m_{1}\left(y_{b}\right)\right]} \\
{\left[h_{2} m_{3}\left(y_{a}\right)-h_{1} m_{1}\left(y_{a}\right)\right]=\left[h_{2} m_{3}\left(y_{b}\right)-h_{1} m_{1}\left(y_{b}\right)\right]}
\end{array}\right.\right.
$$

Eqs. (29) can be rewritten as:

$$
\left\{\begin{array}{l}
{\left[m_{2}\left(y_{a}\right)-m_{2}\left(y_{b}\right)\right] / g_{1}=\left[m_{1}\left(y_{a}\right)-m_{1}\left(y_{b}\right)\right] / g_{2}} \\
{\left[m_{3}\left(y_{a}\right)-m_{3}\left(y_{b}\right)\right] / h_{1}=\left[m_{1}\left(y_{a}\right)-m_{1}\left(y_{b}\right)\right] / h_{2}}
\end{array}\right.
$$

As $g_{1}, g_{2}$ are coprime and $h_{1}, h_{2}$ are coprime too, Eqs.(30) must be equivalent to the following:

$$
\left\{\begin{array}{l}
{\left[m_{2}\left(y_{a}\right)-m_{2}\left(y_{b}\right)\right]=n_{1} g_{1},\left[m_{1}\left(y_{a}\right)-m_{1}\left(y_{b}\right)\right]=n_{1} g_{2}} \\
{\left[m_{3}\left(y_{a}\right)-m_{3}\left(y_{b}\right)\right]=n_{2} h_{1},\left[m_{1}\left(y_{a}\right)-m_{1}\left(y_{b}\right)\right]=n_{2} h_{2}}
\end{array}\right.
$$

Where $n_{1}, n_{2}$ are integers and $n_{1}, n_{2} \neq 0$. From Eqs.(31) we know:

$$
n_{1} g_{2}=n_{2} h_{2}
$$

Note that $k_{3}$ be the g.c.m. of $\lambda_{2}$ and $\lambda_{3}$, and the g.c.m. of $\lambda_{1}, \lambda_{2}$ and $\lambda_{3}$ not exists, so, $n_{1} g_{2}=n_{1} k_{3} q_{1}, n_{2} h_{2}=n_{2} k_{3} q_{2}$, where $q_{1}, q_{2}$ are positive integers which are coprime. Eq.(32) can be rewritten as follow:

$$
n_{1} q_{1}=n_{2} q_{2}
$$

We have $n_{1}=q_{2}, n_{2}=q_{1}$, then: 
$\left[m_{1}\left(y_{a}\right)-m_{1}\left(y_{b}\right)\right]=n_{1} g_{2}=q_{2} g_{2}=\left(h_{2} / k_{3}\right) g_{2}=\lambda_{2} \lambda_{3} /\left(k_{1} k_{2} k_{3}\right)$ In the similar way,

$\left[m_{2}\left(y_{a}\right)-m_{2}\left(y_{b}\right)\right]=n_{1} g_{1}=\lambda_{1} \lambda_{3} /\left(k_{1} k_{2} k_{3}\right),\left[m_{3}\left(y_{a}\right)-m_{3}\left(y_{b}\right)\right]=n_{2} h_{1}=\lambda_{1} \lambda_{2} /\left(k_{1} k_{2} k_{3}\right)$. Combining inequations

(12) (14), we have: $\quad-R / \lambda_{1}<\left[m_{1}\left(y_{a}\right)-m_{1}\left(y_{b}\right)\right]<R / \lambda_{1},-R / \lambda_{2}<\left[m_{2}\left(y_{a}\right)-m_{2}\left(y_{b}\right)\right]<R / \lambda_{2}$ and

$-R / \lambda_{3}<\left[m_{3}(y)-m_{3}(y)\right]<R / \lambda_{3}$, which means $-R / \lambda_{1}<\lambda_{2} \lambda_{3} /\left(k_{1} k_{2} k_{3}\right)<R / \lambda_{1},-R / \lambda_{2}<\lambda_{1} \lambda_{3} /\left(k_{1} k_{2} k_{3}\right)<R / \lambda_{2}$ and $-R / \lambda_{3}<\lambda_{1} \lambda_{2} /\left(k_{1} k_{2} k_{3}\right)<R / \lambda_{3}$. Hence, Eqs. (23) will hold when $R \leq \lambda_{1} \lambda_{2} \lambda_{3} /\left(k_{1} k_{2} k_{3}\right)$.

From the analysis above, there exists an unique mapping from $\left(m_{1}(y), m_{2}(y), m_{3}(y)\right)$ to $\left[\lambda_{1} \varphi_{1}(y)-\lambda_{2} \varphi_{2}(y)\right] / 2 \pi,\left[\lambda_{1} \varphi_{1}(y)-\lambda_{3} \varphi_{3}(y)\right] / 2 \pi$ and $\left[\lambda_{2} \varphi_{2}(y)-\lambda_{3} \varphi_{3}(y)\right] / 2 \pi$ (i.e., $m_{2}(y) \lambda_{2}-m_{1}(y) \lambda_{1}$, $\left.m_{3}(y) \lambda_{3}-m_{1}(y) \lambda_{1}, m_{3}(y) \lambda_{3}-m_{2}(y) \lambda_{2}\right)$ when $R \leq \lambda_{1} \lambda_{2} \lambda_{3} /\left(k^{2} k_{1} k_{2} k_{3}\right)$, where $R$ is the resolution of projector, and $k$ is the g.c.m. of $\lambda_{1}, \lambda_{2}$ and $\lambda_{3}, \lambda_{1}=k g_{1}, \lambda_{2}=k g_{2}, \lambda_{3}=k g_{3}$, where $k_{1}$ is the g.c.m. of $g_{1}$ and $g_{2}, k_{2}$ is the g.c.m. of $g_{1}$ and $g_{3}, k_{3}$ is the g.c.m. of $g_{2}$ and $g_{3}$. 
Table 1. Mapping from $m_{2}(y) \lambda_{2}-m_{1}(y) \lambda_{1}, m_{3}(y) \lambda_{3}-m_{1}(y) \lambda_{1}$ and $m_{3}(y) \lambda_{3}-m_{2}(y) \lambda_{2}$ to $m_{1}(y), m_{2}(y), m_{3}(y)$

when $\lambda_{1}=54, \lambda_{2}=72, \lambda_{3}=90, R=1024$

\begin{tabular}{|c|c|c|c|c|c|}
\hline$m_{1}(y)$ & $m_{2}(y)$ & $m_{3}(y)$ & $m_{2}(y) \lambda_{2}-m_{1}(y) \lambda_{1}$ & $m_{3}(y) \lambda_{3}-m_{1}(y) \lambda_{1}$ & $m_{3}(y) \lambda_{3}-m_{2}(y) \lambda_{2}$ \\
\hline 1 & 0 & 0 & -54 & -54 & 0 \\
\hline 5 & 3 & 3 & -54 & 0 & 54 \\
\hline 9 & 6 & 5 & -54 & -36 & 18 \\
\hline 13 & 9 & 7 & -54 & $\begin{array}{l}-72 \\
\end{array}$ & -18 \\
\hline 17 & 12 & 10 & -54 & -18 & 36 \\
\hline 2 & 1 & 1 & -36 & -18 & 18 \\
\hline 6 & 4 & 3 & -36 & -54 & -18 \\
\hline 10 & 7 & 6 & -36 & 0 & 36 \\
\hline 14 & 10 & 8 & -36 & -36 & 0 \\
\hline 18 & 13 & 10 & -36 & -72 & -36 \\
\hline 18 & 13 & 11 & -36 & 18 & 54 \\
\hline 3 & 2 & 1 & -18 & -72 & -54 \\
\hline 3 & 2 & 2 & -18 & 18 & 36 \\
\hline 7 & 5 & 4 & -18 & -18 & 0 \\
\hline 11 & 8 & 6 & -18 & -54 & -36 \\
\hline 11 & 8 & 7 & -18 & 36 & 54 \\
\hline 15 & 11 & 9 & -18 & 0 & 18 \\
\hline 0 & 0 & 0 & 0 & 0 & 0 \\
\hline 4 & 3 & 2 & 0 & -36 & -36 \\
\hline 8 & 6 & 4 & 0 & -72 & -72 \\
\hline 8 & 6 & 5 & 0 & 18 & 18 \\
\hline 12 & 9 & 7 & 0 & -18 & -18 \\
\hline 16 & 12 & 9 & 0 & -54 & -54 \\
\hline 16 & 12 & 10 & 0 & 36 & 36 \\
\hline 1 & 1 & 0 & 18 & -54 & -72 \\
\hline 1 & 1 & 1 & 18 & 36 & 18 \\
\hline 5 & 4 & 3 & 18 & 0 & -18 \\
\hline 9 & 7 & 5 & 18 & -36 & -54 \\
\hline 13 & 10 & 8 & 18 & 18 & 0 \\
\hline 17 & 13 & 10 & 18 & -18 & -36 \\
\hline 2 & 2 & 1 & 36 & -18 & -54 \\
\hline 6 & 5 & 4 & 36 & 36 & 0 \\
\hline 10 & 8 & 6 & 36 & 0 & -36 \\
\hline 14 & 11 & 8 & 36 & -36 & -72 \\
\hline 18 & 14 & 11 & 36 & 18 & -18 \\
\hline
\end{tabular}


Figure 1. The photograph of the toy model

Figure 2. Experiment results when $\left(\lambda_{1}, \lambda_{2}, \lambda_{3}\right)=(54,72,90)$. (a), (b) and (c) are the deformed fringe patterns; (d), (e) and (f) are the wrapped phase maps get by three-step PSP

Figure 3. Experiment results when $\left(\lambda_{1}, \lambda_{2}, \lambda_{3}\right)=(54,72,90)$. (a) is the designed shadow noise filter; (b) is the recovered absolute phase map of $\lambda=54$.

Figure 4. Experiment results when $\left(\lambda_{1}, \lambda_{2}, \lambda_{3}\right)=(25,30,35)$. (a), (b) and (c) are the deformed fringe patterns; (d), (e) and (f) are the wrapped phase maps get by four-step PSP.

Figure 5. Experiment results when $\left(\lambda_{1}, \lambda_{2}, \lambda_{3}\right)=(25,30,35)$. (a) is the designed shadow noise filter; (b) is the recovered absolute phase map of $\lambda=25$.(c) is the absolute phase map of $\lambda=25$ which has been removed the carrier-plane.

Figure 6 . The 3D view of the recovered object. 


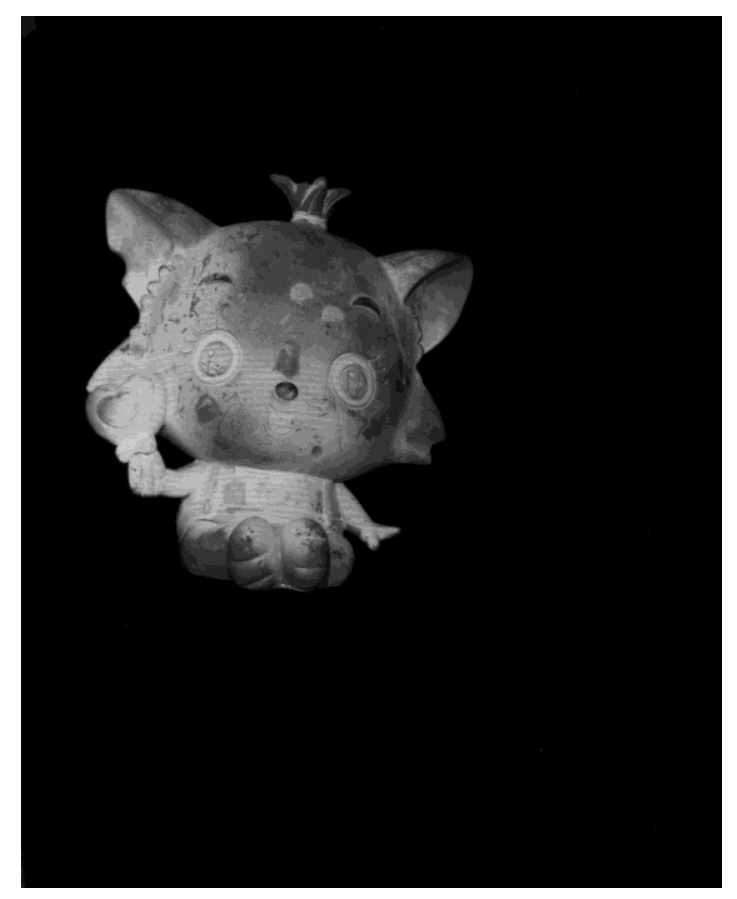

Fig。 1

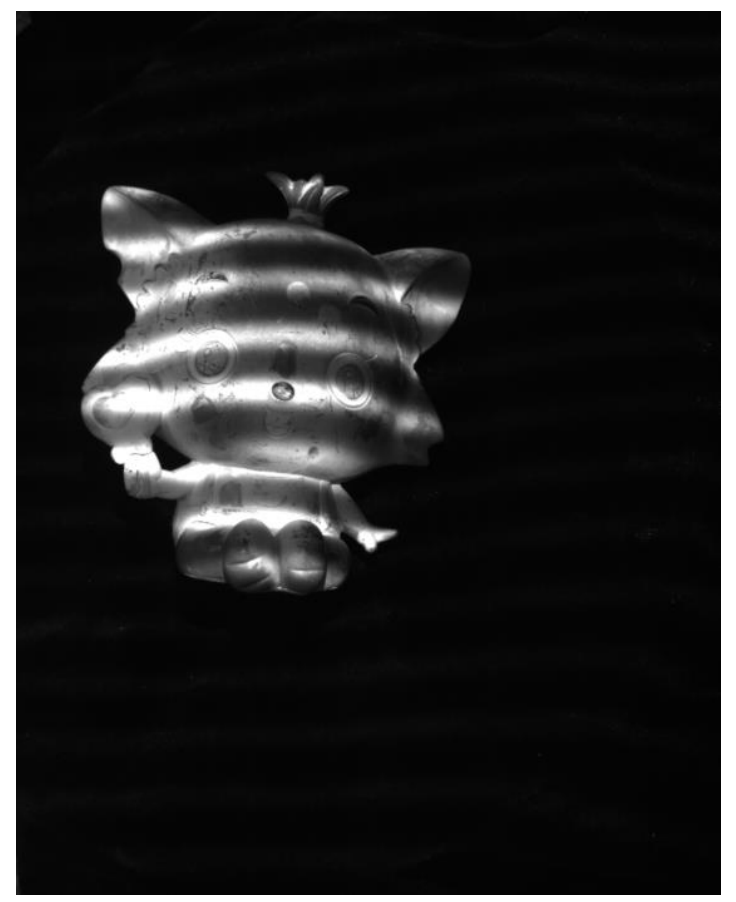

Fig。2(a) 


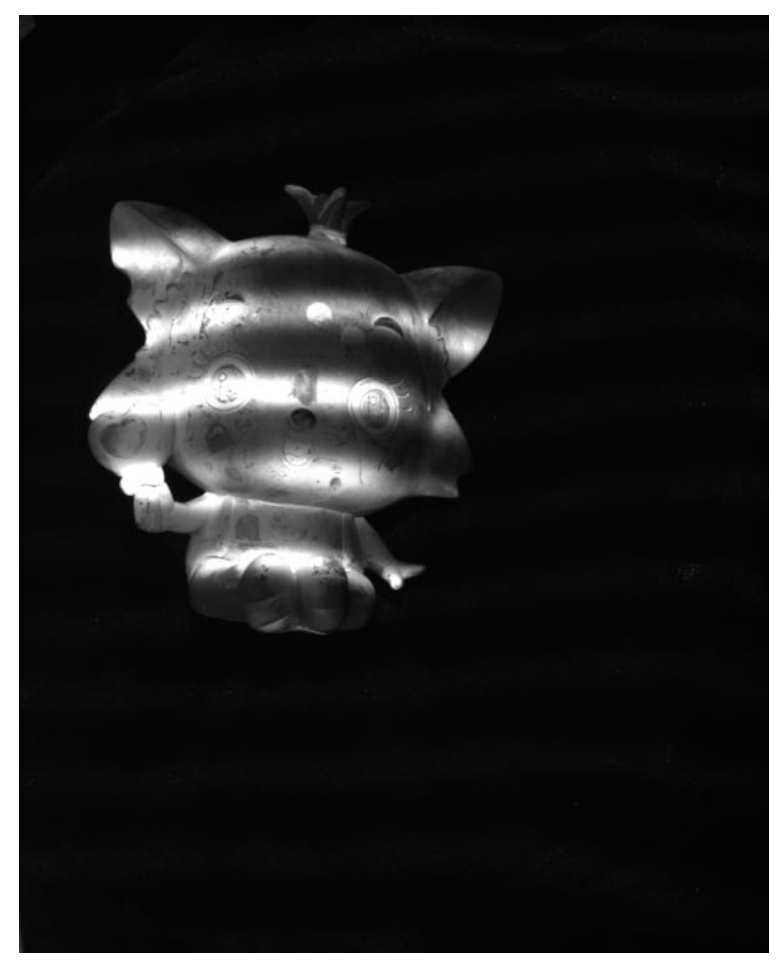

Fig.2(b)

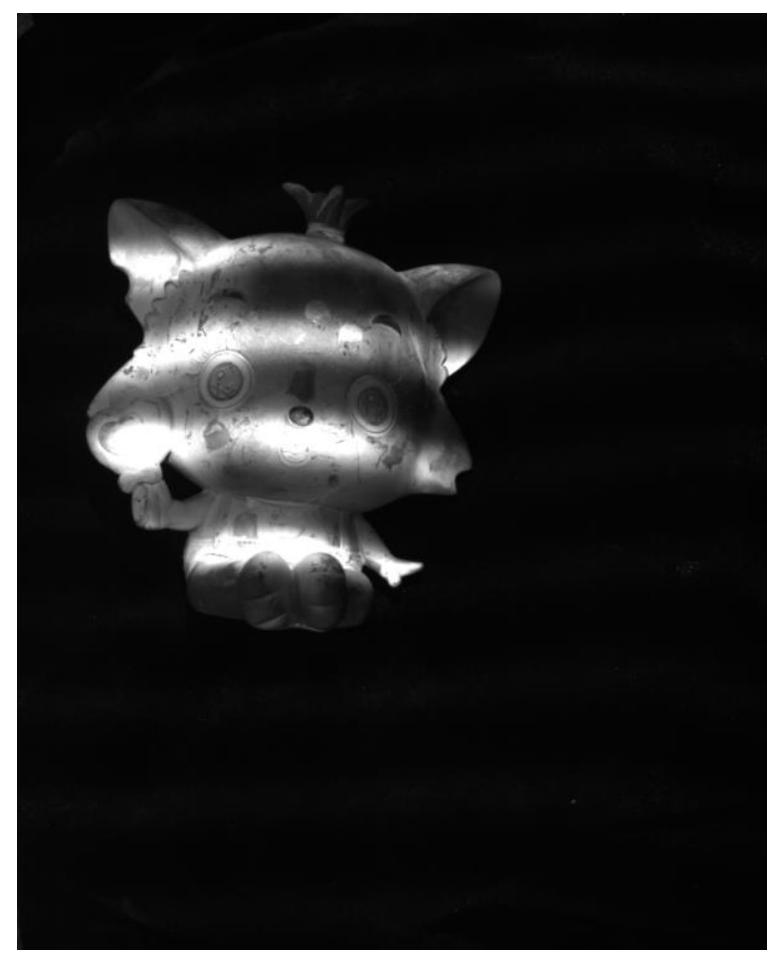

Fig.2(c) 


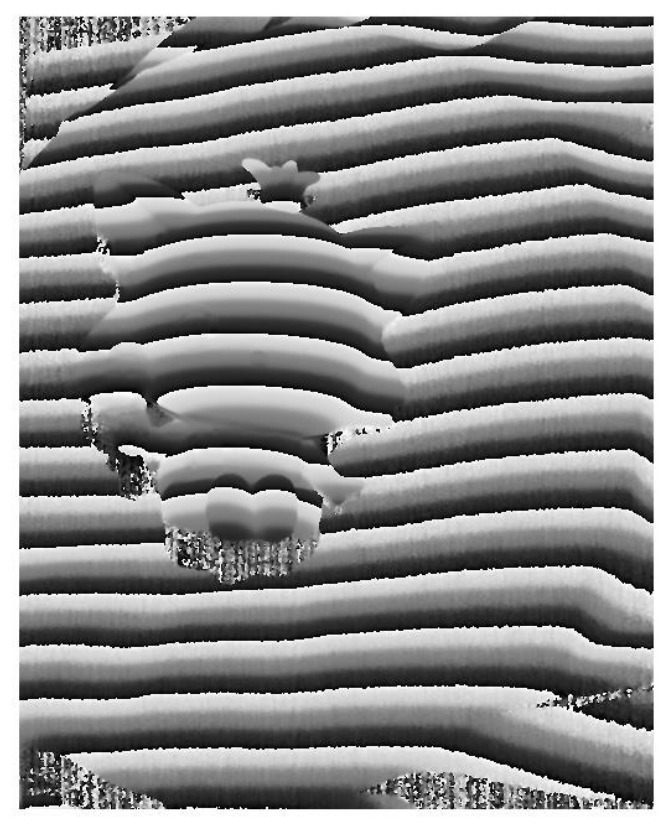

Fig.2(d)

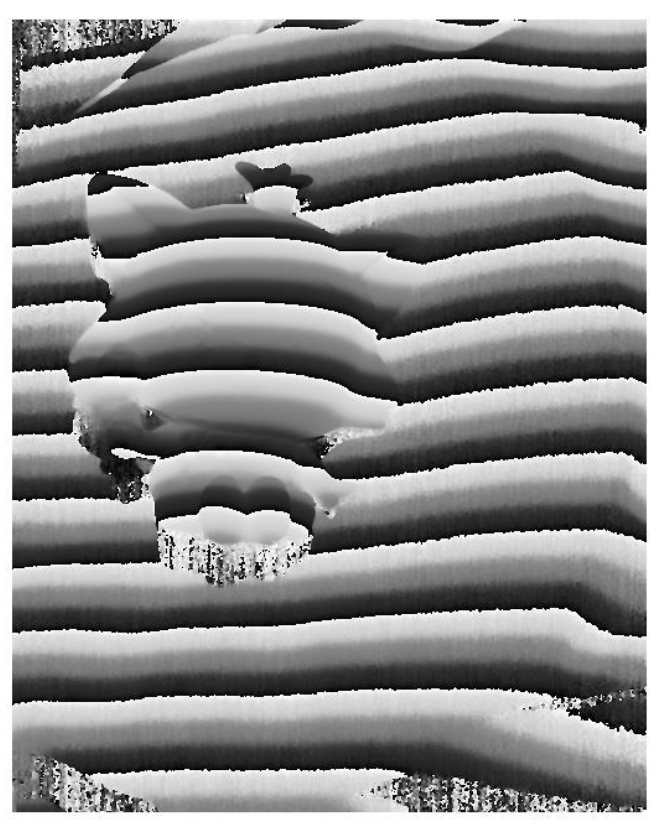

Fig.2(e) 


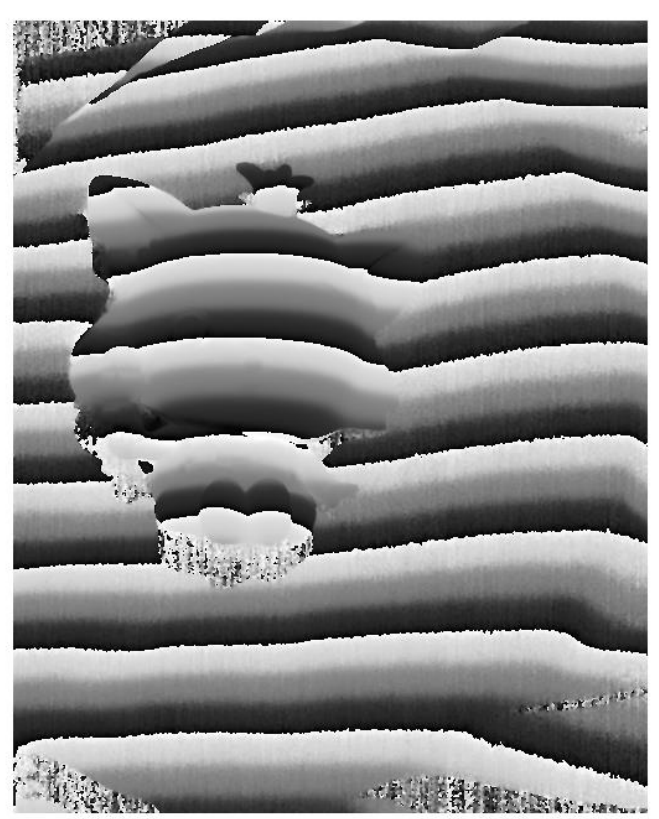

Fig.2(f)

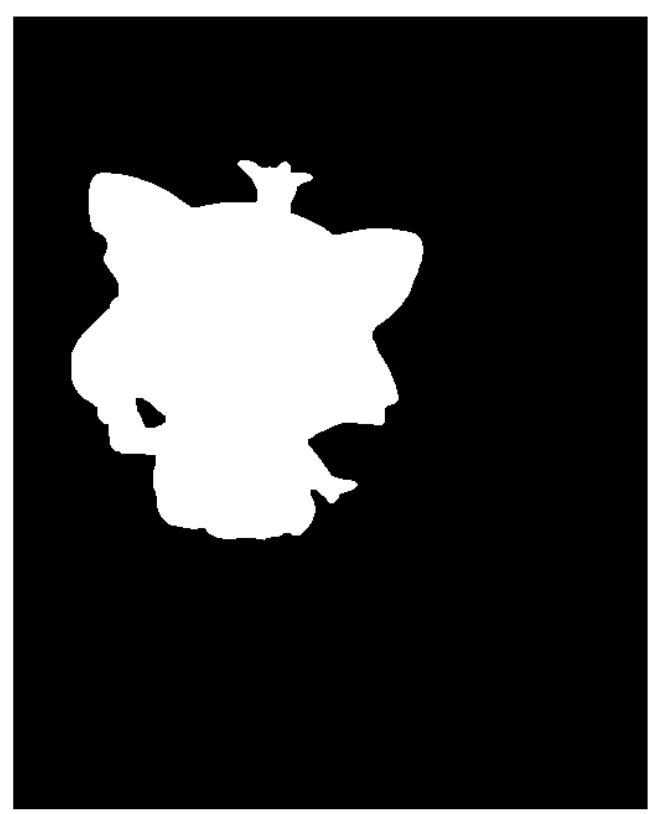

Fig.3(a) 


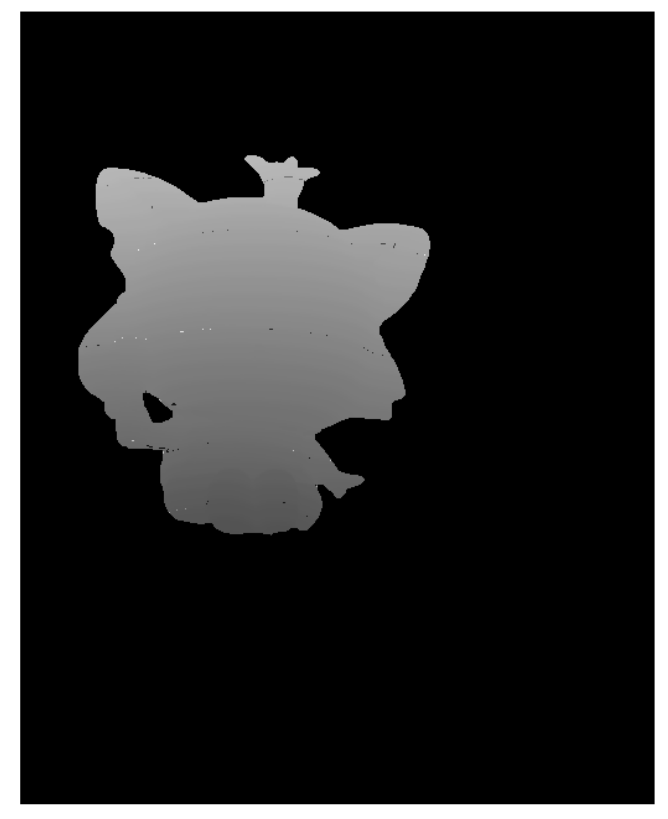

Fig.3(b)

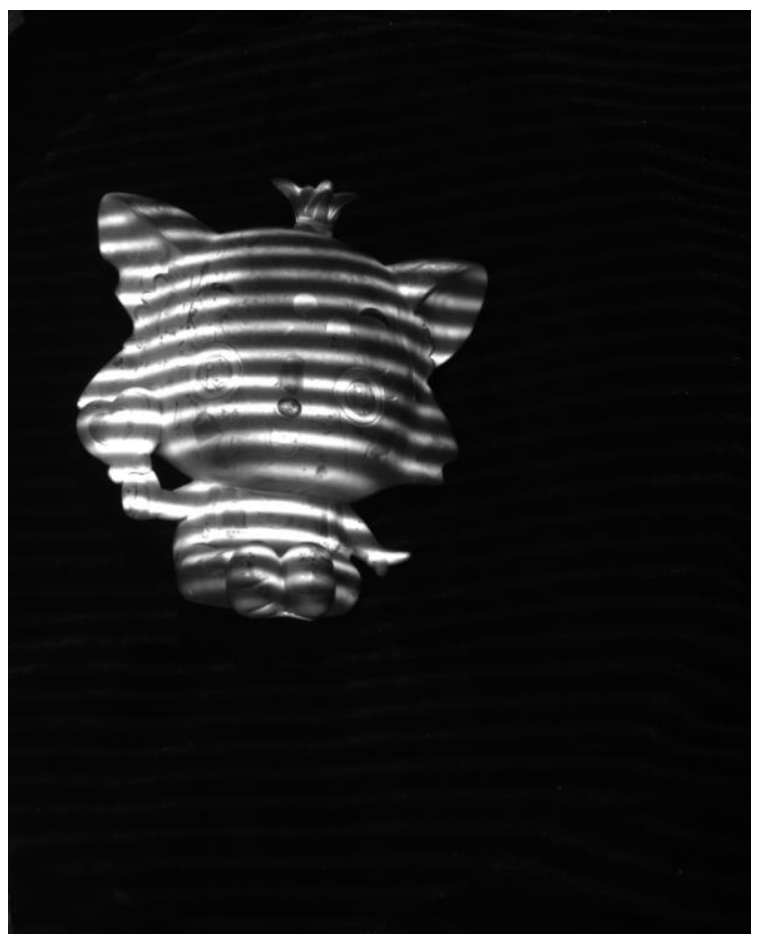

Fig.4(a) 


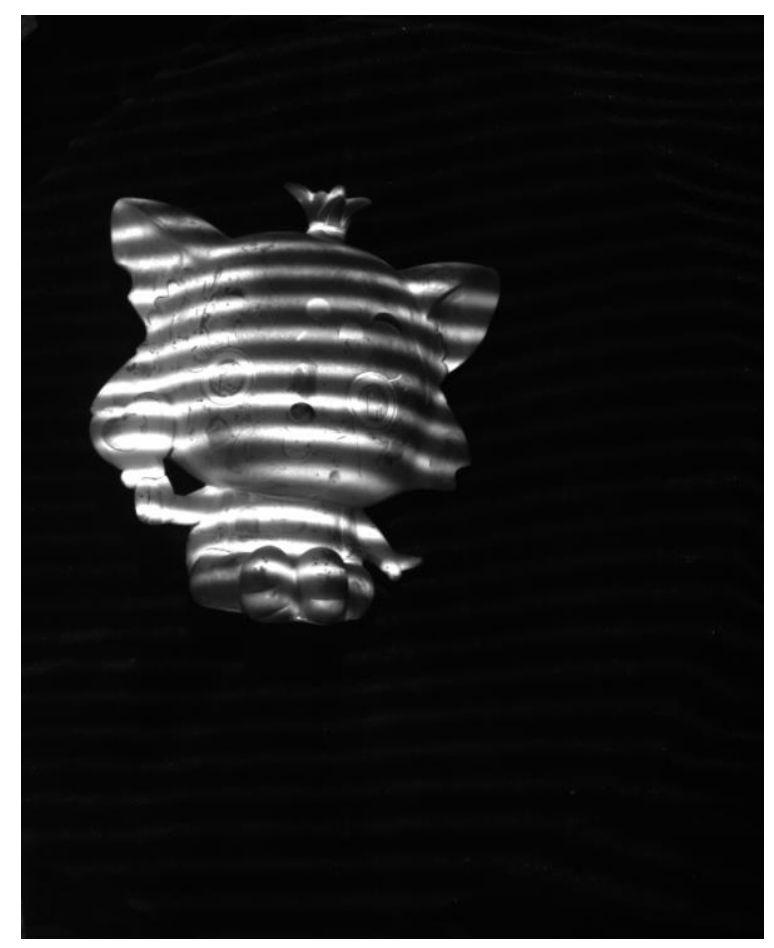

Fig.4(b)

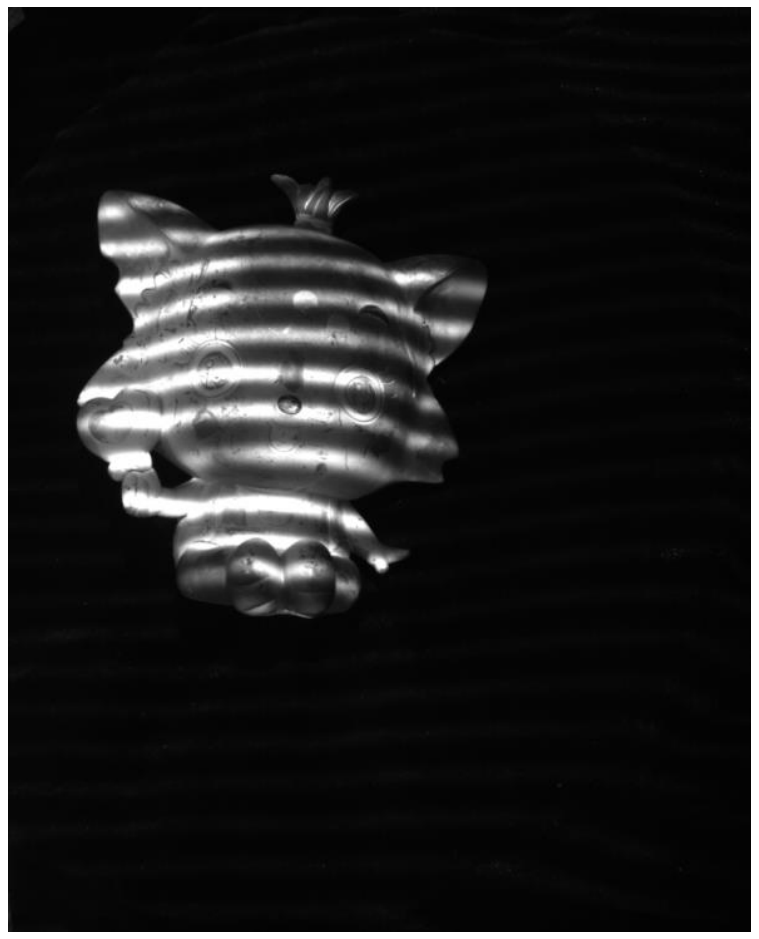

Fig.4(c) 


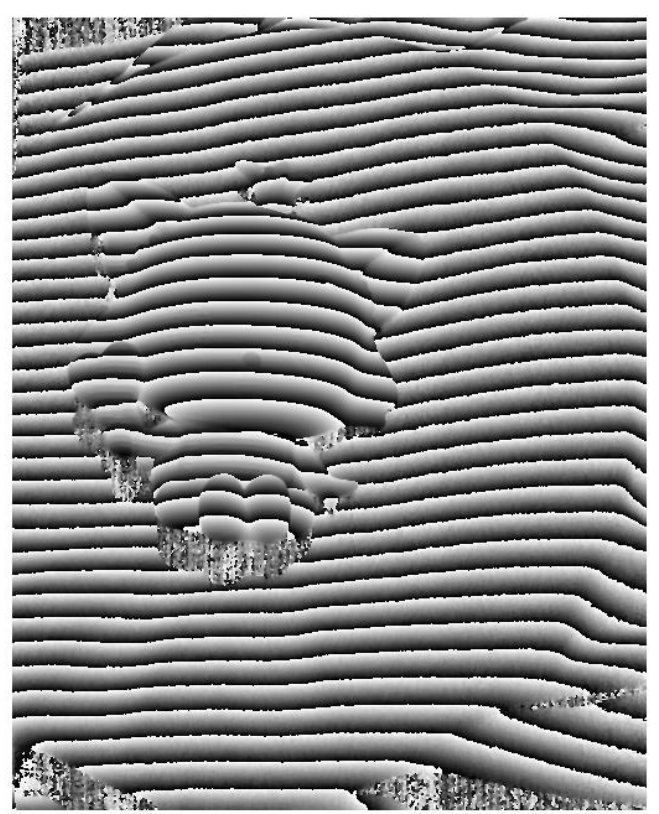

Fig.4(d)

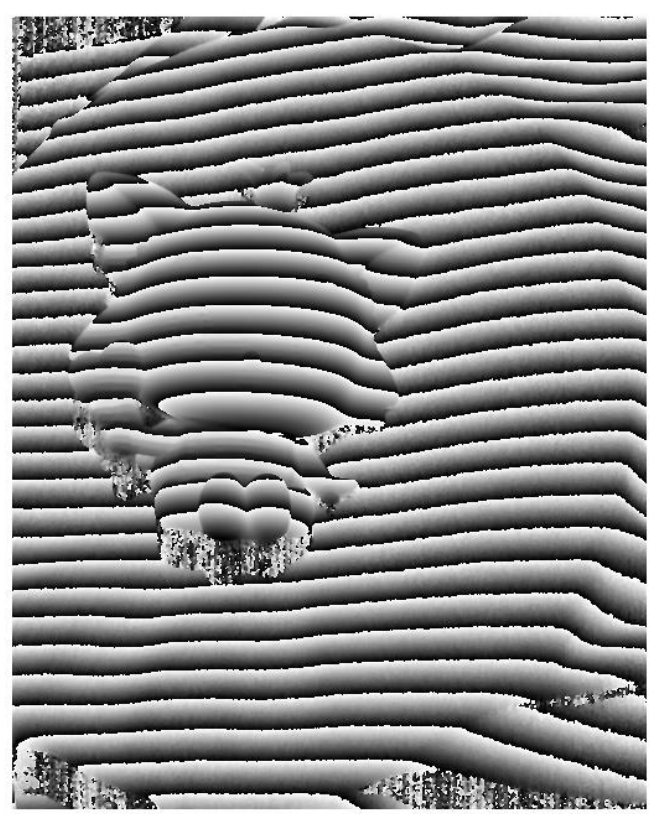

Fig.4(e) 


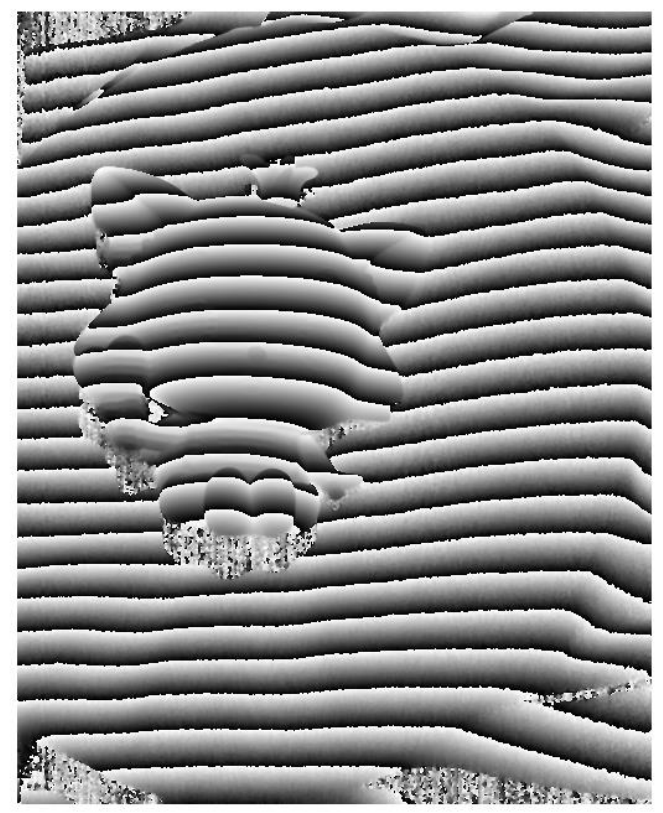

Fig.4(f)

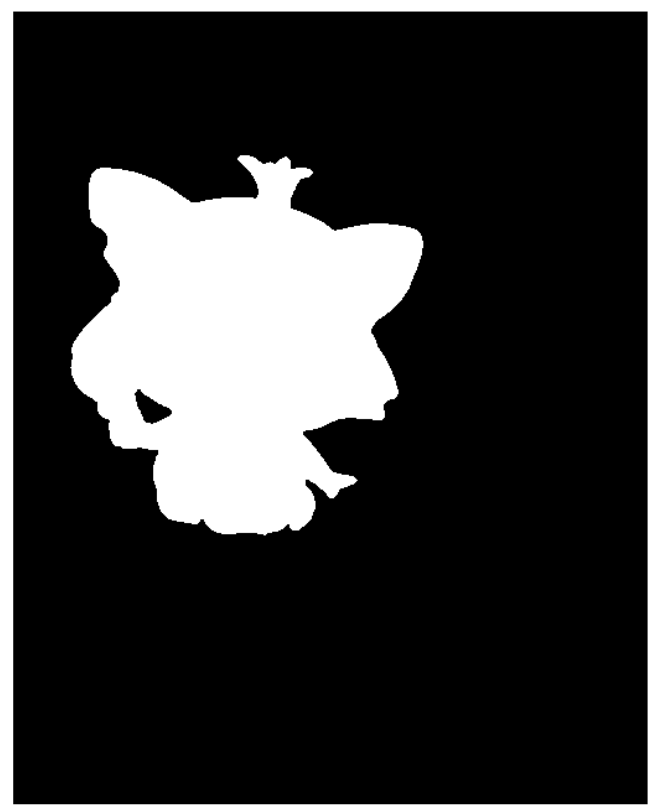

Fig.5(a) 


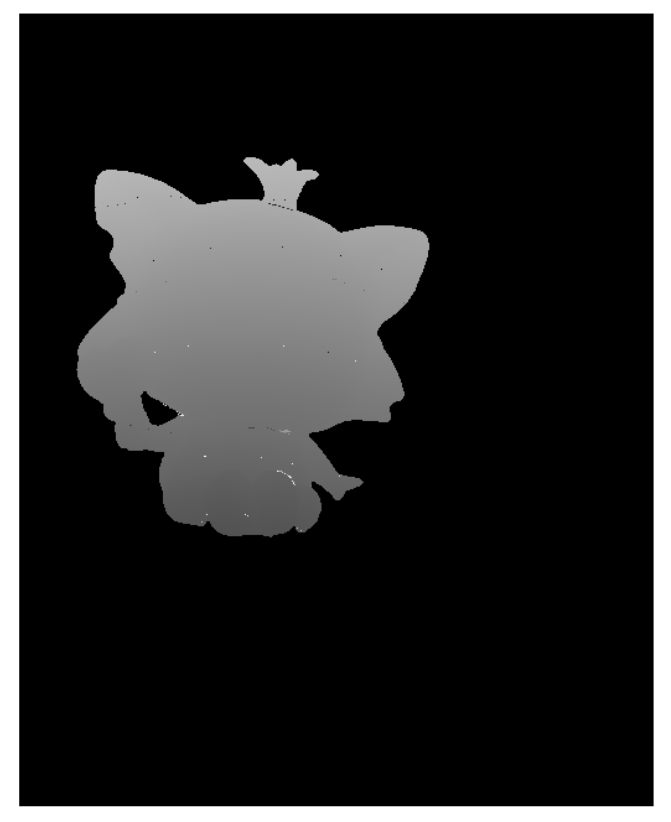

Fig.5(b)

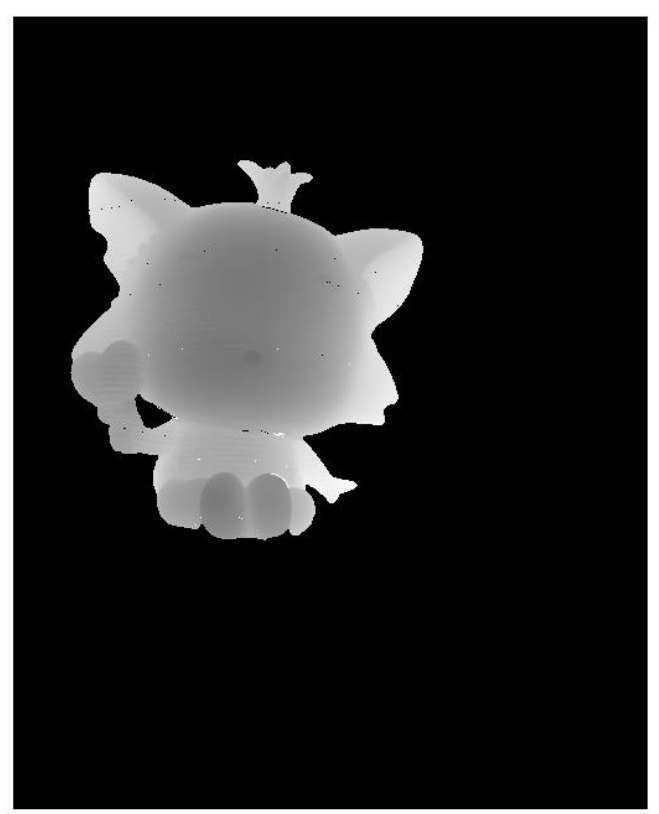

Fig.5(c) 


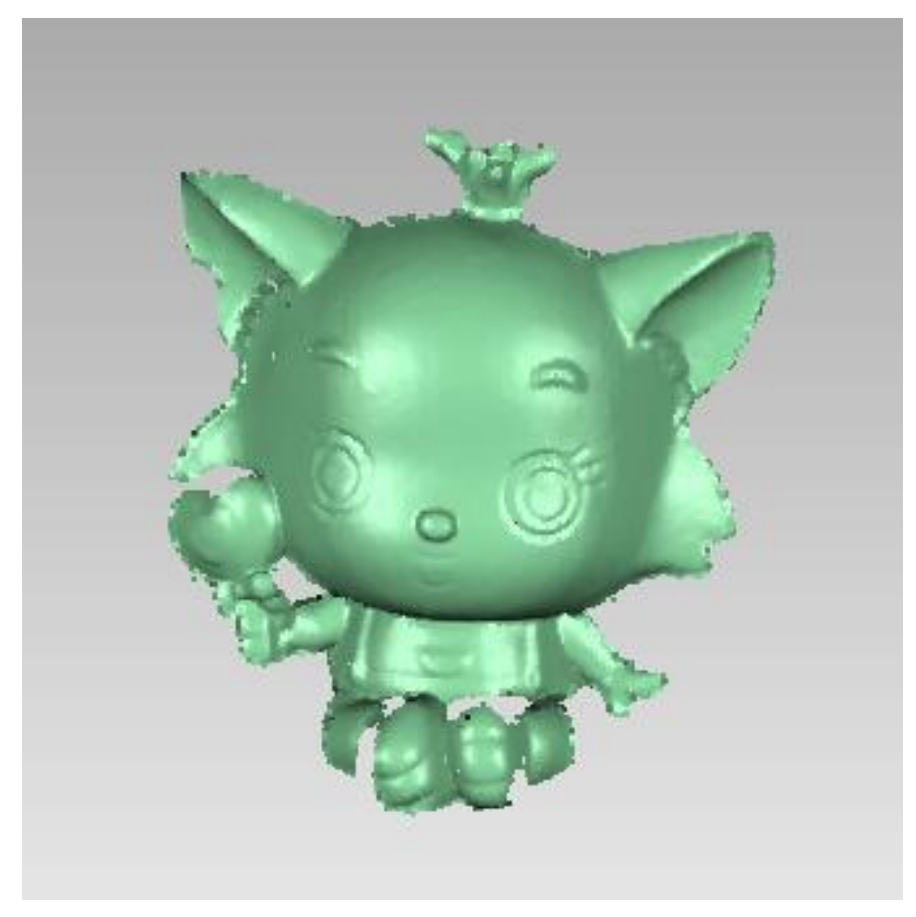

Fig.6 